\title{
Single High-Dose Intravenous Immunoglobulin Therapy for Kawasaki Disease Increases Plasma Viscosity
}

\author{
Reizo Baba, MD; Atsuko Shibata, MD; Masahito Tsurusawa, MD
}

\begin{abstract}
Background Intravenous immunoglobulin therapy, widely used for various autoimmune and systemic inflammatory diseases including Kawasaki disease (KD), is occasionally associated with thromboembolic adverse effects caused by an abrupt increase in blood viscosity. Scarce information is available, however, regarding the effect of single high-dose immunoglobulin therapy for KD on blood viscosity.

Methods and Results Eleven boys and 5 girls (mean age: 2.1 years) with acute-phase KD underwent single high-dose immunoglobulin therapy. Plasma viscosity before the treatment was 1.18 centipoises $(\mathrm{SD}=0.06)$, but it significantly rose to 1.34 centipoises $(\mathrm{SD}=0.06)(\mathrm{p}<0.001)$. Multiple regression analysis revealed that, among various factors including hematocrit, plasma concentrations of total protein, immunoglobulin $\mathrm{G}$ ( $\operatorname{IgG}$ ), immunoglobulin A ( $\operatorname{IgA})$, and immunoglobulin $\mathrm{M}(\operatorname{IgM})$, only plasma $\operatorname{IgG}$ concentration was included in the model to explain plasma viscosity $\left(\mathrm{R}^{2}=0.59, \mathrm{p}<0.001\right)$.

Conclusions Single high-dose regimen for acute-phase KD increases blood viscosity and therefore might increase the risk of thromboembolism. (Circ J 2005; 69: 962-964)
\end{abstract}

Key Words: Kawasaki disease; Globulin; Viscosity

$\mathbf{K}$ awasaki disease $(\mathrm{KD})$ is an acute febrile vasculitis of unknown etiology that occurs predominantly in infants and young children. Although acute signs and symptoms of this disease are self-limiting, coronary arterial ectasia sometimes develops, which might lead to myocardial infarction, sudden cardiac death, or ischemic heart diseases! !-3 $^{-1}$

The most reliable treatment for acute-phase KD is the combination of aspirin and high-dose intravenous immunoglobulin (IVIg) therapy4 However, IVIg therapy especially for elderly patients who have concomitant atherosclerosis is occasionally associated with serious thromboembolism such as myocardial infarction, ${ }^{5-7}$ pulmonary thromboembolism, stroke,-11 and venous thrombosis! $!^{12-15}$ Although several mechanisms are proposed to explain the prothrombotic effects of IVIg11 the major cause is considered to be an increase in blood viscosity! 6 A report by Nishikawa et al shows that IVIg therapy for KD did not increase whole blood viscosity! ${ }^{17}$ However, they used a 5-day regimen (400 mg $\cdot \mathrm{kg}^{-1} \cdot \mathrm{day}^{-1}$ for 5 days) instead of a single large dose method that had been shown to be more effective and the standard treatment at that time. The single high-dose regimen and subsequent high infusion rate might be related with a higher incidence of thromboembolism ${ }^{13}$ Unfortunately, we have scarce information regarding the effect of single high-dose immunoglobulin therapy for $\mathrm{KD}$ on the morbidity of thromboembolism or blood viscosity.

Therefore, the aim of the present study was to elucidate the effect of single high-dose IVIg therapy for KD on

(Received March 15, 2005; revised manuscript received April 26, 2005; accepted May 11, 2005)

Department of Paediatrics, Aichi Medical University School of Medicine, Aichi, Japan

Mailing address: Reizo Baba, MD, Department of Paediatrics, Aichi Medical University School of Medicine, 21-Karimata-Yazako, Nagakute, Aichi, Aichi 480-1195, Japan. E-mail: babar@aichi-med-u. ac.jp plasma viscosity.

\section{Methods}

\section{Study Subjects}

The study subjects of the present study were recruited from patients with acute-phase KD who had been treated with IVIg therapy at Aichi Medical University Hospital, Aichi, Japan during the period between January and December 2004. We have confined the use of IVIg in combination with aspirin to patients who had had coronary arterial lesions before defervescence or who were at risk for having coronary arterial aneurisms, ie, having at least 4 or more of the following conditions: (i) white blood cell count more than $12,000 / \mathrm{mm}^{3}$; (ii) platelet counts less than $350,000 / \mathrm{mm}^{3}$; (iii) C-reactive protein more than $4.5 \mathrm{mg} / \mathrm{dl}$; (iv) hematocrit less than $35 \%$; (v) serum albumin less than $3.5 \mathrm{mg} / \mathrm{dl}$; (vi) age under 12 months; and (vii) male sex! ${ }^{18}$ Patients who did not meet these conditions were treated solely with aspirin. Eleven boys and 5 girls met these criteria for IVIg treatment and agreed to participate in the current study. One boy was excluded from the study because he had developed hypothermia with chillness after IVIg had been infused $(0.5 \mathrm{~g} / \mathrm{kg})$. Eventually 10 boys and 5 girls were enrolled in the current study. Their mean age was 2.1 years $(\mathrm{SD}=1.8)$.

The present study was approved by our institutional review board. Informed consent was obtained from each patient's guardian(s).

\section{Treatment}

All subjects were treated with aspirin with an anti-inflammatory dose of $30 \mathrm{mg} / \mathrm{kg}$, which had been initiated prior to administration of IVIg to prevent thrombotic events caused by possible hyperviscosity syndrome. All subjects were treated with IVIg with a single dose of $2 \mathrm{~g} / \mathrm{kg}$ over $24 \mathrm{~h}$. Mean duration from the onset of KD to the initiation of 
Table 1 Changes in Laboratory Data Before and Immediately After Completion of IVIg Treatment

\begin{tabular}{lccc}
\hline \hline & Before IVIg & After IVIg & $p$ value \\
\hline WBC count $\left(\times 10^{3} / \mathrm{mm}^{3}\right)$ & $18.4(4.3)$ & $8.0(4.1)$ & $<0.001$ \\
CRP $(\mathrm{mg} / \mathrm{dl})$ & $10.8(6.5)$ & $7.1(4.6)$ & $<0.01$ \\
Total protein $(\mathrm{g} / \mathrm{dl})$ & $6.1(0.5)$ & $7.3(0.6)$ & $<0.001$ \\
Albumin $(\mathrm{g} / \mathrm{dl})$ & $3.7(0.4)$ & $3.1(0.4)$ & $<0.001$ \\
IgG $(\mathrm{mg} / \mathrm{dl})$ & $670(150)$ & $2,680(430)$ & $<0.001$ \\
IgA $(\mathrm{mg} / \mathrm{dl})$ & $66(35)$ & $76(38)$ & 0.051 \\
IgM $(\mathrm{mg} / \mathrm{dl})$ & $92(36)$ & $103(53)$ & 0.19 \\
Plasma viscosity $(\mathrm{cP})$ & $1.18(0.06)$ & $1.34(0.06)$ & $<0.001$ \\
\hline
\end{tabular}

Data are expressed as mean (SD). IVIg, intravenous immunoglobulin; $W B C$, white blood cell; $C R P, C$-reactive protein; $I g G$, mmunoglobulin $G$; IgA, immunoglobulin A; IgM, immunoglobulin M; cP, centipoises.

IVIg treatment was 5.1 days $(\mathrm{SD}=1.9)$. Three patients needed additional administrations of IVIg $(2 \mathrm{~g} / \mathrm{kg})$, all of which were effective but one who had transient dilatation of the left coronary artery.

\section{Measurements}

Blood samples $(9 \mathrm{ml})$ were drawn from the peripheral veins immediately before administration and within $12 \mathrm{~h}$ after the completion of IVIg therapy. The whole-blood samples $(2 \mathrm{ml})$ were processed by ethylenediaminetetraacetic acid and were analyzed for complete blood cell count with a Beckman GEN*S autoanalyzer (Beckman Instruments, Fullerton, CA, USA). The residual blood samples were immediately centrifuged at 2,000 rpm for $10 \mathrm{~min}$ and then separated plasma was frozen at $-40^{\circ} \mathrm{C}$ and stored. We measured plasma viscosity with a Brookfield DV-II + machine, which was calibrated before each measurement. Samples were measured at $37.0^{\circ} \mathrm{C}$ and at a shear rate of $50 \mathrm{rev} / \mathrm{min}$. The viscosity was expressed in centipoises $(\mathrm{cP})$. The upper and lower normal limits of plasma viscosity in infant and children in our institution ( $n=6$, mean age: 2.5 years) were 1.12 and $1.27 \mathrm{cP}$, respectively. We also measured plasma concentrations of total protein, albumin, immunoglobulin $\mathrm{G}(\mathrm{IgG})$, immunoglobulin A ( $\operatorname{Ig} \mathrm{A})$, and immunoglobulin $\mathrm{M}$ (IgM) with a Hitachi 7700 autoanalyzer (Hitachi HighTechnologies, Tokyo, Japan).

\section{Statistics}

Paired t-test was used to analyse the effect of IVIg treatment on various measurements. Multiple stepwise regression analysis was used to analyse the determinants of plasma viscosity. We chose such independent variables as hematocrit, plasma concentrations of total protein, IgG, $\operatorname{Ig} \mathrm{A}$, and IgM. All statistical tests were 2-sided. P-values that were less than 0.05 were considered to indicate statistical significance. Statistical analyses were performed with the Japanese version of SPSS software (ver. 11) (Tokyo, Japan).

\section{Results}

None of our subjects developed any symptoms suggestive of thrombosis. The changes in the laboratory data before and immediately after the completion of IVIg treatment are listed in Table 1. All but 2 subjects showed normal plasma viscosity before treatment (Fig 1). However, after IVIg treatment, all subjects showed increases in viscosity and all but 2 subjects exceeded the normal upper

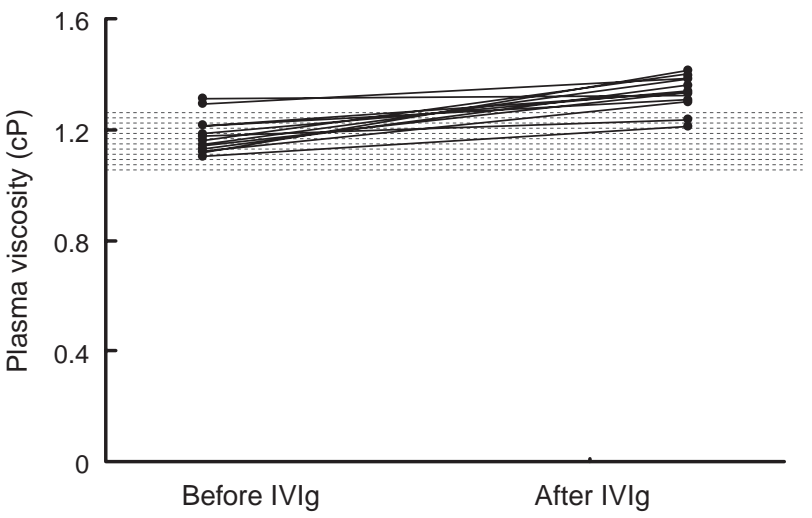

Fig 1. Effect of single high-dose intravenous immunoglobulin infusion on plasma viscosity. Dotted area shows normal values in infants and children. cP, centipoises; IVIg, intravenous immunoglobulin.

limits of our laboratory standards (Fig 1). Multiple regression analysis revealed that only plasma IgG concentration was included in the model to explain plasma viscosity $\left(\mathrm{R}^{2}=0.59, \mathrm{p}<0.001\right)$. Other variables such as hematocrit, plasma concentrations of total protein, $\operatorname{IgA}$, and $\operatorname{IgM}$ were eliminated from the model $(\mathrm{p}=0.853,0.424$, and 0.396 , respectively).

\section{Discussion}

The present study reveals that single high-dose $(2 \mathrm{~g} / \mathrm{kg})$ IVIg treatment for acute-phase KD results in a slight but significant increase in plasma viscosity, which is mainly attributed to the increase in plasma $\mathrm{IgG}$ concentration. Therefore, the emergence of thromboembolic events as a result of an abrupt rise in plasma viscosity must be taken into consideration in treating patients with acute-phase KD with single high-dose IVIg regimen.

Numerous previous studies have shown thromboembolic events are caused by IVIg infusion, especially in elderly people with atherosclerosis - $^{5}$ The morbidity rate of stroke associated with IVIg therapy is reported to be $0.6 \%$ in a series of hospitalized adult patients? Although several mechanisms are proposed to explain the prothrombotic effect, modest elevation in serum viscosity or rapid change in plasma viscosity must play a role? High levels of $\mathrm{IgG}$, immune complex formation, and increased platelet aggregation could increase blood viscosity after IVIg infusion.,9,16 Increased serum viscosity reduces arterial and capillary blood flow, leading to thrombosis $!^{1}$

Generally, small increases in plasma viscosity affect capillary blood flow and the risk of thrombosis sharply increases when serum viscosity exceeds 2 to $3 \mathrm{cP}$ ? This threshold is, however, valid only for subjects with normal circulation? In patients with $\mathrm{KD}$, who have general vasculitis and occasionally coronary arterial aneurisms and hence have limited microcirculatory blood flow in the myocardium, a small increase in blood viscosity can tip the rheology balance and cross symptomatic viscosity threshold precipitating thromboembolic events?,16

In contrast to our results, Nishikawa et al reported that IVIg therapy for KD did not increase whole blood viscosity 17 This discordance might be accounted for by infusion rate. The total doses were the same in both studies, ie, $2 \mathrm{~g} / \mathrm{kg}$. However, Nishikawa et al used a regimen of $400 \mathrm{mg} \cdot \mathrm{kg}^{-1} \cdot$ day $^{-1}$ for 5 consecutive days and we adopted 
a single high-dose method over $24 \mathrm{~h} !^{17}$ Although we did not compare the effect of IVIg infusion regimen on blood viscosity in the present study, single-dose regimens are known to result in higher peak serum IgG levels $!^{19}$ A previous study in accordance with the present study, shows higher levels of serum IgG yield higher plasma viscosity ${ }^{16}$

Although the present study reveals the potential hazard associated with single high-dose IVIg therapy for acutephase $\mathrm{KD}$, we do not have any concrete epidemiological evidence that this regimen actually increases the incidence of thromboembolic adverse effects. The present study did not intend to answer this issue, which might be the major limitation of the study. In addition, few past studies have been published concerning this issue. Newburger et al conducted a large-scale $(n=549)$ multicenter randomized controlled trial and reported no adverse effects suggestive of thromboembolism either in the group who had received IVIg of $2 \mathrm{~g} / \mathrm{kg}$ of body weight or in the group who had IVIg of $400 \mathrm{mg} / \mathrm{kg}$ for 4 consecutive days.$^{19}$ Sato et al also reported that there were no apparent adverse reactions, not only during, but also after IVIg infusion among 72 patients with KD who had received a single $2 \mathrm{~g} / \mathrm{kg}$ dose of IVIg and 73 children who were treated with $400 \mathrm{mg} / \mathrm{kg}$ of body weight per day for 5 consecutive days $2^{20}$ The results of these studies suggest, in spite of numerous epidemiological evidences in adult populations with other underlying diseases, that single high-dose IVIg therapy for acute-phase KD do not at least dramatically increase the incidence of thromboembolic events in comparison with devided regimens, although the number of the subject seems to be insufficient to yield statistically significant differences. It might be explained by the hypothesis that single high-dose IVIg therapy can subside vascular inflammation and thus counterbalance the risk of thromboembolism caused by the increase in blood viscosity.

In summary, single high-dose regimen for acute-phase $\mathrm{KD}$ results in higher blood viscosity and, although there is no epidemiological evidence, might increase the risk of thromboembolism. Therefore, admitting the superiority of single high-dose regimen in reducing the prevalence of coronary arterial lesions, ${ }^{19,21}$ results of the present study raise an alarm in treating acute-phase $\mathrm{KD}$ with single highdose IVIg administration.

\section{References}

1. Newburger JW, Takahashi M, Gerber MA, Gewitz MH, Tani LY, Burns JC, et al. Diagnosis, treatment, and long-term management of Kawasaki disease: A statement for health professionals from the Committee on Rheumatic Fever, Endocarditis and Kawasaki Disease, Council on Cardiovascular Disease in the Young, American Heart Association. Circulation 2004; 110: 2747-2771.

2. Imamura H, Izawa A, Kai R, Yokoseki O, Uchikawa S, Yazaki Y, et al. Trends over the last 20 years in the clinical background of young
Japanese patients with coronary artery disease. Circ J 2004; 68: $186-191$.

3. Onouchi Z, Hamaoka K, Sakata K, Ozawa S, Shiraishi I, Itoi T, et al. Long-term changes in coronary artery aneurysms in patients with Kawasaki disease. Circ J 2005; 69: 265-272.

4. Saji T, Sonobe T, Uemura S, Akagi T, Ayusawa M. Guideline for treatment of acute-phase Kawasaki disease. Acta Paeditrica Cardiologica Japonica 2004; 20: 54-62 (in Japanese).

5. Fruchter O, Blich M, Jacob G. Fatal acute myocardial infarction during severe thrombocytopenia in a patient with idiopathic thrombocytopenic purpura. Am J Med Sci 2002; 323: 279-280.

6. Fisman DN, Smilovitch M. Intravenous immunoglobulin, blood viscosity and myocardial infarction. Can J Cardiol 1997; 13: 775777.

7. Dalakas MC. High-dose intravenous immunoglobulin and serum viscosity: Risk of precipitating thromboembolic events. Neurology 1994; 44: 223-226.

8. Emerson GG, Herndon CN, Sreih AG. Thrombotic complications after intravenous immunoglobulin therapy in two patients. Pharmacotherapy 2002; 22: 1638-1641.

9. Okuda D, Flaster M, Frey J, Sivakumar K. Arterial thrombosis induced by IVIg and its treatment with tPA. Neurology 2003; 60: $1825-1826$.

10. Byrne NP, Henry JC, Herrmann DN, Abdelhalim AN, Shrier DA, Francis CW, et al. Neuropathologic findings in a Guillain-Barre patient with strokes after IVIg therapy. Neurology 2002; 59: 458-461.

11. Caress JB, Cartwright MS, Donofrio PD, Peacock JE Jr. The clinical features of 16 cases of stroke associated with administration of IVIg. Neurology 2003; 60: $1822-1824$.

12. Go RS, Call TG. Deep venous thrombosis of the arm after intravenous immunoglobulin infusion: Case report and literature review of intravenous immunoglobulin-related thrombotic complications. Mayo Clin Proc 2000; 75: 83-85.

13. Evangelou N, Littlewood T, Anslow P, Chapel H. Transverse sinus thrombosis and IVIg treatment: A case report and discussion of riskbenefit assessment for immunoglobulin treatment. J Clin Pathol 2003; 56: $308-309$.

14. Oh KT, Boldt HC, Danis RP. Iatrogenic central retinal vein occlusion and hyperviscosity associated with high-dose intravenous immunoglobulin administration. Am J Ophthalmol 1997; 124: 416-418.

15. Hefer D, Jaloudi M. Thromboembolic events as an emerging adverse effect during high-dose intravenous immunoglobulin therapy in elderly patients: A case report and discussion of the relevant literature. Ann Hematol 2004; 83: 661-665.

16. Rheinhart WH, Berchtold PE. Effect of high-dose intravenous immunoglobulin therapy on blood rheology. Lancet 1992; 339: 662-664.

17. Nishikawa M, Ichiyama T, Hasegawa M, Kawasaki K, Matsubara T, Furukawa S. Safety from thromboembolism using intravenous immunoglobulin therapy in Kawasaki disease: Study of whole-blood viscosity. Pediatr Int 2003; 45: 156-158.

18. Harada K. Intravenous gamma-globulin treatment in Kawasaki disease. Acta Paediatr Jpn 1991; 33: 805-810.

19. Newburger JW, Takahashi M, Beiser AS, Burns JC, Bastian J, Chung KJ, et al. A single intravenous infusion of gamma globulin as compared with four infusions in the treatment of acute Kawasaki syndrome. N Engl J Med 1991; 324: 1633-1639.

20. Sato N, Sugimura T, Akagi T, Yamakawa R, Hashino K, Eto G, et al. Selective high dose gamma-globulin treatment in Kawasaki disease: Assessment of clinical aspects and cost effeciveness. Pediatrics International 1999; 41: 1-7.

21. Durongpisitkul K, Gururaj VJ, Park JM, Martin CF. The prevention of coronary artery aneurysm in Kawasaki disease: A meta-analysis on the efficacy of aspirin and immunoglobulin treatment. Pediatrics 1995; 96: $1057-1061$. 